\title{
La (de)construcción del género en la ficción política: el caso de Borgen
}

\section{The (De)construction of Gender in Political Fiction: The Borgen Case}

\section{RESUMEN}

La presente investigación se centra en la representación de las mujeres políticas en las ficciones seriadas a través de la serie Borgen (2010-2013) y desde una perspectiva de género. Para ello, desde una metodología cualitativa, se realiza una revisión de los estereotipos de género que marcan habitualmente a los personajes de mujeres políticas en la ficción y se comprueba si se reproducen o se rompen en la serie objeto del estudio. Los resultados de la investigación revelan que Borgen presenta una transgresión del género en lo relativo a cuatro ámbitos: conciliación vida pública-privada, rasgos de personalidad, competencia temática y liderazgo, proponiendo así una hoja de ruta para la representación de las mujeres en los medios de comunicación, así como para la realización de una comunicación política desde una perspectiva de género.

Palabras clave: series de televisión, ficción política, mujer política, estereotipos de género, Borgen.

\footnotetext{
Abstract

This investigation focuses, from a gender perspective, on the representation of the female politicians on fiction series, specifically in Borgen (2010-2013). In order to analyze it, a review of the gender stereotypes that habitually characterise female politicians in fiction is carried out through a qualitative methodology. This study also check if the Borgen series keep or destroy these stereotypes. The results of the research demonstrate that Borgen presents a transgression of gender in four different areas: conciliation of the public-private life, personality features, thematic competence and leadership. Therefore the thesis suggests a roadmap for the representation of women in the media, as well as a realization of a political communication from a gender perspective.

Keywords: 1.-Introducción. 2.- El papel socializador de las series de televisión. 3.- La comunicación política en la ficción televisiva: análisis del caso Borgen. 4.- Resultados y discusión. 5.- Referencias.

\section{SUMARIO}

1.-Introducción. 2.- El papel socializador de las series de televisión. 3.- La comunicación política en la ficción televisiva: análisis del caso Borgen. 4.- Resultados y discusión. 5.- Referencias.
} 


\section{1.- Introducción}

Las series de televisión son un producto cultural que ha experimentado una gran transformación en los últimos años. Con la llegada de las plataformas de contenidos audiovisuales y las redes sociales, el consumo de las series se ha modificado en gran medida, «haciendo al usuario partícipe de los contenidos a través de diversas opciones de interacción con los mismos» (Francisco-Lens y Rodríguez Vázquez, 2020, p. 188).

La televisión ya no es el único soporte reservado para la reproducción y difusión de productos audiovisuales, ahora los dispositivos tecnológicos facilitan que estos productos puedan ser consumidos superando las fronteras del tiempo y espacio que imponía la parrilla televisiva. Por esta razón, en la actualidad sería más adecuado utilizar el concepto de «ficciones seriadas» en lugar de «series de televisión» (Carrión Domínguez, 2019).

Entre las ficciones seriadas que han gozado de cierto éxito en los últimos años encontramos un espacio reservado para las series políticas, algunos ejemplos de ello son The Good Wife (2009-2016), Scandal (2012-2018), Veep (2012-2019) o House of Cards (2013-2018), series que han demostrado que el entretenimiento político ocasiona interés y seguimiento masivo alrededor del mundo (Padilla Castillo y Sosa Sánchez, 2018).

Pero no solo han experimentado un auge los contenidos de ficción política; con el avance de las sociedades en materias de igualdad de género, también destaca la producción y el consumo de productos culturales que reflejan la opresión que siguen sufriendo las mujeres a día de hoy, a la vez que proporcionan referentes y modelos de conducta que rompen con los valores tradicionales del sistema patriarcal. Esto ha provocado que las mujeres en las series hayan pasado «de ser las novias, hijas, esposas, amantes de los personajes masculinos, a ser las protagonistas por derecho propio» (Vélez, 2020, p.6).

Los efectos de la aparición y el establecimiento de las nuevas tecnologías, por lo tanto, «han permitido romper con el sesgo de género de la información institucionalizada, aumentar la participación y la diversidad de la mirada social, poner en conexión a las mujeres como colectivo y promover una alta difusión del activismo y sus estrategias» (Marín Ramos, 2019, p. 28).

En esta intersección entre dramas políticos y temáticas feministas se encuentra Borgen (2010-2013), una serie que se convirtió en un fenómeno de masas en numerosos países y que ha marcado el camino que luego han seguido otras series en materia de igualdad de género y representación de la política. Tras estrenarse en Dinamarca el 26 de septiembre de 2010, «fue distribuida, por orden cronológico, a Noruega, Suecia, Finlandia, Estados Unidos, Holanda, Reino Unido, Corea del Sur, Francia, Alemania, Japón, España, Grecia, Italia, Polonia, Serbia y Rusia, entre otros» (Padilla Castillo y Sosa Sánchez, 2018, p.88). En el contexto danés también fue todo un éxito: «Borgen atrajo a alrededor de 1,5 millones de espectadores por episodio, según las estadísticas de medidores de televisión de TNS Gallup: esto equivale al $27 \%$ de la población danesa, un total de 5,6 millones en ese momento» (Boukes, Aalbers y Andersen, 2020, p.4). 
Borgen tiene como protagonista principal a una mujer política, Birgitte Nyborg (interpretada por Sidse Babett Knudsen), que ejerce el papel de la primera mujer en llegar al cargo de Primera Ministra de Dinamarca. La manera en que concibe y lleva a cabo la política Birgitte Nyborg, así como las relaciones que se producen en su ámbito laboral y familiar, rompen con el modo tradicional de representar la política y a las mujeres políticas en televisión.

Dado que los productos culturales como series y películas tienen un gran poder a la hora de conformar identidades, tanto de forma individual como colectiva, no podemos ignorar el valor desde una perspectiva de género que tiene una serie como Borgen en lo relativo a la representación de mujeres en política y también a la realización de una comunicación política con perspectiva de género.

Para observar de qué manera esta serie presenta una disrupción con respecto a lo hecho hasta el momento, se pretende analizar cómo se representa a los personajes de la serie, haciendo especial hincapié en la protagonista, Birgitte Nyborg, y comprobar si se reproducen o no estereotipos de género en lo relativo a cuatro ámbitos: vida privada y vida pública, rasgos de personalidad, competencia temática y liderazgo.

\section{2.- El papel socializador de las series}

Los medios de comunicación constituyen una herramienta esencial en la construcción, reproducción y difusión de significados. Esto cobra especial importancia en el contexto actual en el que la política se desarrolla en gran medida en el ámbito mediático. Es decir, «los medios de comunicación de masas no son los depositarios del poder, pero en conjunto constituyen el espacio en el que se decide el poder» (Castells, 2008, p.4).

Es importante recalcar que los medios de comunicación han devenido uno de los grupos socializadores de mayor relevancia debido a varias razones: son capaces de transcender los límites de la capacidad humana de comunicación, sustituyen actividades sociales, se integran en nuestra vida cotidiana y, por último, los demás agentes de la sociedad se adaptan a la lógica mediática (Lois y Alonso, 2014).

Teniendo en cuenta que «los productos televisivos contribuyen, de forma cotidiana y más o menos sistemática, a generar identidades a partir de los mecanismos narrativos, semióticos e interpelativos que se ponen en marcha en cada acto de significación» (Belmonte y Guillamón, 2008, p. 116), y que, además, en la actualidad se está dando la conocida como «edad de oro de las ficciones seriadas» (Carrión Domínguez, 2019, p.44)., no se puede ignorar el gran poder que las series pueden llegar a tener en el proceso de socialización de las personas.

Este trabajo pretende observar cómo se representa el género en las series, entendiendo que se trata de productos culturales ampliamente consumidos y capaces de construir identidades, tanto de forma individual como colectiva, proporcionando modelos, valores y significados, así como contribuyendo a normalizarlos. Como apunta Elena Galán (2007, p. 236), «las series de ficción, además de servir de entretenimiento, presentan modelos de identificación que son imitados y que tienden a fomentar y a enraizar, aún más, representaciones estereotipadas». 
De esta manera se fomenta el caldo de cultivo perfecto «para que exista y perdure un sistema de sometimiento y dominio de un sexo (el masculino) sobre el otro (el femenino)», y esto «no sólo puede basarse en la coerción física, es necesario que exista una desigualdad de género basada en el ámbito de lo simbólico» (Hormigos Vaquero y Ballester Buigues, 2020).

Tanto las series como las películas han constituido herramientas de construcción del género dado que reproducen una visión estereotipada de la realidad, asociando a sus personajes atributos arquetípicos de la feminidad o de la masculinidad en función de su sexo (Sierra Caballero, 2017). No obstante, las series de televisión también han servido para dar visibilidad a temas que atañen a las mujeres pero que hasta no hace mucho tiempo habían sido invisibles en la televisión, como la violencia machista, la homosexualidad, la inmigración o la conciliación laboral y familiar, entre otros:

\begin{abstract}
Las series también han sido testigo de la notable evolución de la mujer en el terreno social y de su incorporación al mundo laboral de un modo masivo. Esta transformación ha sido consecuentemente reflejada por la ficción, aunque tal y como se ha demostrado en gran parte de las ocasiones, las representaciones de género siguen siendo fieles a las convenciones. Los estereotipos de género están tan interiorizados en nuestra cultura que se transmiten a menudo de un modo indirecto y precisan análisis profundos y elaborados para poder ser detectados, corregidos y adaptados a las nuevas circunstancias sociales (Galán Fajardo, 2007: 236).
\end{abstract}

Pero de la misma manera que las series de televisión proporcionan una visión sesgada y arquetípica de la realidad, también pueden fomentar y difundir todo lo contrario: modelos de conducta que rompan con los estereotipos de género tradicionales.

Por esta razón se ha elegido la serie Borgen como objeto del presente estudio, pues, en el caso de la representación de la mujer política en la pequeña pantalla, esta serie constituye una hoja de ruta en cuanto a la ruptura de estereotipos y a la forma de llevar a cabo una comunicación política con perspectiva de género.

Para averiguar de qué manera las ficciones seriadas pueden difundir paradigmas igualitarios, resulta indispensable poner el foco en los estereotipos de género que se reproducen o se rompen en esta serie. Los estereotipos de género constituyen una serie de ideas generalizadas atribuidas tradicionalmente a las mujeres y a los hombres, y que estos asumen durante su proceso de socialización. Dado que reproducen una lógica patriarcal, «tienen como consecuencia la desigualdad entre los sexos y se convierten en agentes de discriminación, impidiendo el pleno desarrollo de las potencialidades y las oportunidades de ser de cada persona» (Varela, 2005, p. 325).

Los medios de comunicación se han convertido en una herramienta muy potente de reproducción y difusión de los estereotipos de género. El recurso a estereotipos en comunicación facilita el mismo proceso comunicativo, pues devienen atajos mentales que aportan fluidez, condensan información y evocan imágenes socialmente compartidas. 
Cabe dejar patente cuáles son los estereotipos más habituales que suelen marcar la representación mediática de las mujeres en general, y de las mujeres políticas en particular, para así poder determinar si Borgen rompe con los mismos y, en caso afirmativo, establecer de qué manera se produce esta ruptura y en qué medida.

\begin{tabular}{|c|c|}
\hline ÁMBITO & ESTEREOTIPOS \\
\hline \multirow{4}{*}{ Conciliación vida pública y privada } & $\begin{array}{l}\text { 1. La programación televisiva hace énfasis más en la vida } \\
\text { privada y romántica de las mujeres que en su vida pública } \\
\text { o profesional. }\end{array}$ \\
\hline & $\begin{array}{l}\text { 2. Las mujeres tienen casi el doble de probabilidad de in- } \\
\text { terpretar el papel de esposas en comparación con las veces } \\
\text { que los varones representan el de maridos. }\end{array}$ \\
\hline & $\begin{array}{l}\text { 3. Con referencia al matrimonio, la televisión muestra in- } \\
\text { directamente que el matrimonio daña a los hombres y es } \\
\text { beneficioso para las mujeres. }\end{array}$ \\
\hline & $\begin{array}{l}\text { 4. Cuando una mujer logra salir de los roles que la encasillan } \\
\text { en la vida privada e interpreta un papel profesional o direc- } \\
\text { tivo, son estadísticamente significativos los casos en los que } \\
\text { la trama muestra que tiene deterioros éticos o emocionales. }\end{array}$ \\
\hline \multirow[t]{2}{*}{ Rasgos de personalidad } & $\begin{array}{l}\text { 5. A los hombres se les adjudica el talento, la racionalidad, } \\
\text { la estabilidad y el poder. Otros rasgos estereotipadamen- } \\
\text { te masculinos: agresivo, amante del peligro, atlético, líder, } \\
\text { duro, individualista, personalidad fuerte, egoísta. }\end{array}$ \\
\hline & $\begin{array}{l}\text { 6. Mientras que a las mujeres se les confiere el atractivo, la } \\
\text { ternura y la pasividad. Otros rasgos estereotipadamente } \\
\text { femeninos: sumisa, llora fácilmente, tierna, amante de los } \\
\text { niños, cariñosa, afectuosa, comprensiva y sensible a las ne- } \\
\text { cesidades de los otros. }\end{array}$ \\
\hline \multirow[t]{2}{*}{ Competencia temática } & $\begin{array}{l}\text { 7. Normalmente se asocia a las mujeres con los llamados } \\
\text { temas compasivos (compassion issues) o temas femeninos } \\
\text { (feminine issues) que son los temas relacionados con la lu- } \\
\text { cha contra la pobreza, la educación, el arte, el cuidado de } \\
\text { la salud. }\end{array}$ \\
\hline & $\begin{array}{l}\text { 8. Se considera que los hombres son más competentes en } \\
\text { los llamados temas masculinos (masculine issues) que inclu- } \\
\text { yen cuestiones de economía, empleo, comercio, defensa, } \\
\text { asuntos exteriores, control de armas, entre otros. }\end{array}$ \\
\hline \multirow{3}{*}{ Liderazgo } & $\begin{array}{l}\text { 9. Las características consideradas como masculinas son } \\
\text { asociadas con el liderazgo. }\end{array}$ \\
\hline & $\begin{array}{l}\text { 10. Pero, si son las mujeres las que presentan estas caracte- } \\
\text { rísticas, son penalizadas por parecer demasiado «masculi- } \\
\text { nas». Por ejemplo, la ambición en el hombre es vista como } \\
\text { un rasgo de liderazgo mientras que en una mujer suele es- } \\
\text { tar asociada con connotaciones negativas. }\end{array}$ \\
\hline & $\begin{array}{l}\text { 11. El liderazgo de las mujeres políticas va a menudo parejo } \\
\text { al apoyo de un hombre, siendo definidas por su relación } \\
\text { con un mentor varón. }\end{array}$ \\
\hline
\end{tabular}

Fuente: elaboración a partir de los análisis de Virginia García Beaudoux (2014), Nuria Fernández (2014) y Elena Galán Fajardo (2007). 


\section{3.- La comunicación política en la ficción televisiva: análisis del caso Borgen}

La serie Borgen se emitió por primera vez en Dinamarca el 26 de septiembre de 2010 y su último capítulo fue emitido el 10 de marzo de 2013. Esta ficción política fue creada por Adam Price y escrita por Jeppe Gjerving Gram y Tobias Lindholm, y su trama se desarrolla a lo largo de 30 capítulos divididos en 3 temporadas de 10 capítulos cada una.

Tras su estreno, Borgen fue distribuida a una gran cantidad de países (Noruega, Suecia, Finlandia, Estados Unidos, Holanda, Reino Unido, Corea del Sur, Francia, Alemania, Japón, España, Grecia, Italia, Polonia, Serbia, Rusia, etc.) llegando en poco tiempo a convertirse en un fenómeno de masas (Padilla Castillo y Sosa Sánchez, 2018).

El título de la serie se corresponde con la denominación coloquial con la que los daneses se refieren al Palacio de Christianborg, donde se concentran los tres poderes del Estado danés. Este edificio alberga el Parlamento, la oficina del Primer Ministro y la Corte Suprema, así como distintos espacios reservados a la monarquía, como el Salón del Trono o las caballerizas reales.

La serie retrata el ascenso de Birgitte Nyborg al puesto de Primera Ministra, siendo la primera mujer danesa en ocupar este puesto, así como las dificultades que debe afrontar una vez alcanzado el poder. Sin embargo, no solo se centra en las vicisitudes que atañen a la actividad política de la protagonista, sino también pone el foco la dificultad para compaginar el cargo con su vida personal y familiar, mostrando así una realidad que sigue afectando en la actualidad a las mujeres cuyo trabajo no les permite llevar a cabo una conciliación real.

Cobra especial relevancia también la representación de las relaciones que se dan entre el poder político y los medios de comunicación, mostrando de esta manera la complicada lucha por dominar el relato y la opinión pública. Dada la importancia que se le da en la serie a estas relaciones entre el sistema mediático y el político, Borgen deviene un objeto idóneo de análisis desde el punto de vista de la comunicación política, así como de la perspectiva de género aplicada a las ficciones seriadas.

Este estudio se va a centrar principalmente en la figura y actividad de Birgitte Nyborg, pero también en su relación con los demás personajes con protagonismo esencial. Para ello se estudiará la estereotipación de género presente en la serie comprobando la ruptura o reproducción de los estereotipos anteriormente clasificados, haciendo hincapié en los personajes y capítulos relevantes en este respecto, y atendiendo a los cuatro ámbitos mencionados:

Vida pública y privada:

En Borgen no se hace énfasis más en la vida privada y romántica de las mujeres que en su vida pública o profesional, pues la trama gira principalmente en torno a la actividad política de la protagonista como Primera Ministra de Dinamarca. También cobra relevancia su vida privada, pero la representación que se hace de esta consigue fomentar nuevos modelos de organización familiar. En concreto cabe 
hacer hincapié en el acuerdo al que llegan Birgitte Nyborg y su marido, Phillip Christensen, y que se hace patente desde el primer capítulo de la serie, un acuerdo basado en la alternancia, durante cinco años, de la responsabilidad principal de los cuidados y las tareas del hogar.

Cuando Birgitte Nyborg llega a Primera Ministra, es su marido el encargado de realizar las labores domésticas y de cuidados de los hijos que tienen en común, dejando en un segundo plano su carrera profesional para que Birgitte no tenga que renunciar a su carrera política.

Respecto al estereotipo referente a que «las mujeres tienen casi el doble de probabilidad de interpretar el papel de esposas en comparación con las veces que los varones representan el de maridos», cabe apuntar que esto no solo ocurre en las series de televisión, también en la realidad política y mediática podemos observar cómo las mujeres quedan repetidamente relegadas al papel de «esposas de», siendo obviada su formación, preparación o incluso su nombre. También es bastante común, sobre todo en países como Estados Unidos, ver a las esposas de los políticos acompañarles como fieles compañeras en acontecimientos importantes. En cambio, no solemos ver a los hombres bajo el tratamiento de «maridos de».

En Borgen, sin embargo, Phillip Christensen es quien acompaña a Birgitte Nyborg, quien interpreta el papel de «marido de la Primera Ministra», pero la verdadera protagonista es ella. Podemos verlo claramente en múltiples ocasiones a lo largo de la serie, por ejemplo al inicio cuando, el día de las elecciones generales, llegan al Palacio de Christianborg y él ocupa un segundo plano, de simple acompañante, de apoyo de Birgitte Nyborg.

Por otro lado, en lo referente al estereotipo que afirma que «en la televisión se muestra indirectamente que el matrimonio daña a los hombres y es beneficioso para las mujeres» cabe señalar que, esta afirmación, extraída del análisis realizado por Virginia García Beadoux, hace referencia a la comparación entre los logros obtenidos por los personajes masculinos casados y solteros, y las mujeres casadas y solteras. Este estudio concluye que «los varones tienen mayor probabilidad de tener éxito (en el marco de esta investigación se define el éxito como el logro de los objetivos propuestos y el fracaso como no lograrlos) si son solteros: los hombres solteros fracasan 32 veces y los casados 45 por cada 100 que tienen éxito. En cambio, las mujeres tienen más probabilidades de ser exitosas si están casadas: la proporción de fracasos es 29 si están casadas y 42 si son solteras, de cada 100 éxitos» (García Beadoux, 2014, p. 53).

Teniendo en cuenta esto, y observando el caso que nos ocupa, podríamos afirmar que este estereotipo sí que se reproduce en la serie, pues Phillip Christensen no logra evolucionar en su vida profesional, se ve obligado a renunciar a ascensos y mejores condiciones laborales (capítulo 10, temporada 1) y esto lo lleva a acarrear con una fuerte frustración que acaba repercutiendo en su vida personal y, por supuesto, en su matrimonio.

Si observamos la segunda parte de este estereotipo, que el matrimonio «es beneficioso para las mujeres», podemos observar que también se cumple. Cuando están casados, Birgitte llega a ser la Primera Ministra de Dinamarca y, según está 
establecido en el acuerdo que tienen entre ambos, Phillip es quien se encarga de los cuidados y renuncia en lo profesional, mientras Birgitte se centra en su carrera política. Por ello podemos deducir que quien sale beneficiada es ella, mientras él es quien sale perjudicado.

Es importante remarcar que el matrimonio perjudica a Phillip Christensen en lo personal (porque pasa mucho tiempo solo en casa con sus hijos y Birgitte nunca está, siempre está trabajando y porque se siente frustrado por tener que renunciar en su vida laboral), y en lo laboral (rechaza ascensos y mejoras de condiciones por priorizar las labores domésticas y de cuidados, así como por no perjudicar la imagen de su mujer como Primera Ministra, pues no puede aceptar un puesto directivo en una empresa que se va a beneficiar de una ley llevada a cabo por el gobierno de Nyborg). De esta manera, podemos ver un claro cambio de roles de género, siendo el hombre quien sufre las consecuencias de ejercer el rol que ha sido tradicionalmente asociado a las mujeres (quedarse en casa, encargarse de los cuidados, verse obligadas a renunciar en lo laboral...).

Y en cuanto al último estereotipo analizado respecto a la vida pública-privada, que se refiere a que «cuando una mujer logra salir de los roles que la encasillan en la vida privada y consigue interpretar un papel profesional o directivo, son estadísticamente significativos los casos en los que la trama muestra que tiene deterioros éticos o emocionales», podemos afirmar que tampoco se da en la serie.

Birgitte Nyborg no está exenta de cometer errores, ni como Primera Ministra de Dinamarca, ni tampoco como madre y esposa. La serie retrata esto claramente, sobre todo en la segunda temporada podemos ver cómo el centrarse tanto en sus labores presidenciales acaba siendo el motivo que destruye su matrimonio y acaba afectando también a sus hijos. Birgitte Nyborg se niega a aceptar el divorcio, así como a que Phillip Christensen rehaga su vida, pero esta obcecación también le impide ver que su hija está atravesando graves episodios de ansiedad.

La integridad de Birgitte, a pesar de los obstáculos, no se desmorona en ninguno de los dos planos mencionados (vida personal y vida política). La trama no nos muestra a una mujer cegada por el poder, que quiere mantenerse en la presidencia a toda costa o que cae en las garras de la corrupción y la mala praxis política, ni tampoco pone en marcha maniobras para destruir la relación de su ex marido con su nueva novia.

La estrategia política de Nyborg se basa desde el primer momento en las negociaciones, la transparencia y el consenso. Rechaza tajantemente el juego sucio en política, incluso cuando dispone de herramientas que podrían poner en jaque a sus rivales políticos. Por ejemplo, en el primer capítulo (donde se representa el debate electoral) llegan a sus manos facturas del que es aún Primer Ministro (Lars Hesselboe, del Partido Liberal) que demuestran que ha realizado pagos personales con la tarjeta oficial. Cuando su asesor le propone filtrar estos datos a la prensa, Birgitte responde: «Jamás podría volver a mirarme al espejo si llegara al poder de este modo». Tras esto, despide a su jefe de campaña, Kasper Juul, cuando se entera de que ha pasado esta información a Michael Laugesen, líder del Partido Laborista.

También es importante destacar que, cuando se equivoca en sus decisiones 
políticas, es capaz de reconocer su error y pedir disculpas, tanto a su electorado como a los demás políticos. Esto lo podemos ver, por ejemplo, en el capítulo 5 de la segunda temporada cuando, tras una etapa turbulenta para el gobierno (suicidio del Ministro de Exteriores y la dimisión del líder del ministro de medioambiente y líder del Partido Verde, que trae también el abandono de este partido de la coalición de Gobierno), Birgitte pide perdón al líder del Partido Verde y le reconoce que fue su gabinete quien filtró cierta información a la prensa para reconducir la actitud de dicho ministro, pues pensaba que se estaba centrando más en la fama que en su acción política. Hechos como este hacen que el espectador empatice fácilmente con la protagonista, quien no se libra de cometer errores, pero que sabe afrontarlos y pedir disculpas cuando es necesario.

- $\quad$ Rasgos de personalidad

En este apartado se va a tratar de identificar qué rasgos de personalidad estereotípicos aparecen o se transgreden en la representación de algunos personajes principales de la serie. Sin embargo, en lugar de ir uno por uno, como en el grupo de estereotipos anterior, se va a realizar un perfil de cada uno de los personajes que se consideran relevantes en este aspecto.

En esta serie hay algunos personajes que transgreden los rasgos arquetípicos ligados al género, y otros que, en cambio, los reproducen. Es comprensible que ocurra esto, pues una serie que refleja la realidad no puede obviar que en la actualidad aún no se han superado ciertos aspectos que se siguen ligando ineludiblemente a hombres o mujeres. Los personajes que, en cambio, logran mostrar una personalidad que rompe estos estereotipos son, sin embargo, más importantes en la tarea de retransmisión de diferentes modelos de conducta que no se circunscriben a los mandatos de género. Pero no solo que aparezcan estos personajes es importante, sino también la forma en que se presentan, pues si la trama mostrara de forma peyorativa estas actitudes, también se estarían perpetuando los estereotipos ya mencionados.

\section{Birgitte Nyborg:}

Por un lado, podemos afirmar que el personaje de Birgitte Nyborg transgrede la mayoría de estos roles de género. Para empezar, es quien posee y ejerce el poder en la serie, no es una marioneta movida por alguien en la sombra, ni tampoco ocupa un segundo lugar con respecto a un hombre que ostenta un cargo superior. Birgitte Nyborg se deja aconsejar, pero toma las decisiones de forma individual. Un ejemplo lo vemos en el capítulo 2 de la segunda temporada, cuando Birgitte le responde a su jefe de prensa: «Discuto muchas de mis decisiones contigo, Kasper, pero no necesariamente todas».

El personaje de Birgitte Nyborg no se representa de forma objetualizada, ni se explota su belleza física. Tampoco se muestra como una persona sumisa, débil o llorona, sino todo lo contrario: es una política capaz, comprometida y profesional, 
que no duda en dar un golpe en la mesa cuando la situación lo requiere. Un ejemplo muy claro de esto es cuando decide nombrar a Jakob Kruse como comisario europeo cuando se entera de que le ha ocultado información muy importante para postularse en secreto como candidato a la secretaría general del Partido Moderado, con la intención de librarse de él mandándolo a Bruselas (esta parte de la trama es la que da nombre al capítulo 2 de la temporada 2: «En Bruselas nadie puede oírte gritar»).

Birgitte Nyborg muestra una actitud negociadora, de escucha y respeto hacia los demás partidos, incluso cuando no están de acuerdo entre sí. Esto lo podemos ver en las repetidas ocasiones en las que se intentan llevar a cabo acuerdos entre las diferentes formaciones que conforman la coalición de gobierno. Esto sí que podríamos considerarlo como comportamientos que se adscriben y se esperan de las mujeres en política.

Por otra parte, no la podemos considerar como una persona que se deja llevar por sus emociones, aunque sí que es cierto que sus discursos están marcados por un aporte más emocional. De esta forma consigue humanizarse ante el electorado y mostrar una imagen natural que hace que se reciba como una candidata cercana y auténtica. Mientras que Birgitte Nyborg sí que se representa como una mujer cariñosa, a la que le gustan los niños, afectuosa y comprometida con las necesidades de los demás, su personaje no reproduce el rol de sumisa, llorona, tierna.

Tras este análisis podemos afirmar, pues, que el personaje de Birgitte Nyborg logra romper con gran parte de los estereotipos de género en cuanto a rasgos de personalidad que suelen marcar a las mujeres en las ficciones televisivas.

\section{Katrine Fønsmark:}

Es interesante comentar el perfil del personaje de Katrine Fønsmark, la periodista que sigue de cerca a Nyborg durante toda la serie, ya que ejerce un papel relevante en la trama y también interesante desde el punto de vista de género.

Desde el principio de la serie el trabajo y el puesto de Katrine Fønsmark son cuestionados por su juventud y aspecto físico. Un ejemplo se da en el segundo capítulo de la primera temporada cuando su ex compañera de trabajo, Hanne Holm, le acusa de haber conseguido que la echaran: «¿Chivarte de mi alcoholismo fue suficiente para conseguir mi trabajo o también tuviste que follarte al jefe de informativos?».

Sin embargo, Katrine no se nos muestra como una trepadora capaz de pisar a los demás para subir de rango a nivel profesional, sino como una periodista comprometida que lucha por buscar la verdad y exponérsela a la ciudadanía. Le apasiona su trabajo y cree en el periodismo y su función social. Podemos ver reflejada esta ética profesional en numerosos ejemplos, como cuando investiga junto a Hanne Holm a su propio jefe, Michael Laugesen, para averiguar si ha tenido algo que ver con un complot organizado contra un político, y dimite cuando descubre que es verdad (capítulo 4 de la segunda temporada).

El personaje de Katrine también sirve para poner encima de la mesa temas que atañen a las mujeres, por ejemplo, cuando aborta al principio de la primera temporada, cuando muestra la presión que siente por ser una mujer soltera, independi- 
ente, que prioriza su vida profesional, y que a la vez se le juzga por no tener pareja e hijos, porque "se le está pasando el arroz» (capítulo 2 de la segunda temporada) o la dificultad de conciliar trabajo y familia, aumentada por ser madre soltera (capítulo 2 de la tercera temporada).

Katrine es una periodista dedicada, adicta al trabajo, fuerte y con las ideas claras. Este personaje no se muestra como sumisa, llorona, tierna, amante de los niños, cariñosa o afectuosa. Sí que es una mujer atractiva que llama la atención de algunos hombres que aparecen en la serie, pero no se representa de forma sexualizada.

Podemos afirmar que el personaje de Katrine Fønsmark también logra transgredir los estereotipos de género respectivos a los rasgos de personalidad que se tratan en este análisis.

\section{Phillip Christensen:}

Como señala Nuria Varela, «la teoría del género no solo se refiere a las mujeres. De igual manera que el género femenino está construido socialmente y es una obligación para todo el sexo femenino, el género masculino también está edificado sobre mandatos exigidos para todos los varones» (Varela, 2005, p.324).

Por esa razón es interesante tratar a Phillip Christensen, marido (en la primera temporada) y ex marido (segunda y tercera temporada) de Birgitte Nyborg, en este análisis, pues muestra también una ruptura bastante clara de muchos de los estereotipos relativos a los rasgos de personalidad.

Teniendo en cuenta que «aunque la familia patriarcal tradicional ha sido sustituida por otros modelos del familia, los hombres no han asumido todavía, en general, de manera corresponsable las tareas domésticas y las actividades de cuidado» (Padilla Castillo y Sosa Sánchez, 2018, p. 91), el rol de Phillip Christensen durante toda la serie contribuye a reforzar el estatus de igualdad de género en la familia. Él se encarga en gran parte de los cuidados y las tareas domésticas, tanto cuando viven juntos como cuando se divorcian.

Es un hombre atento, dulce y cariñoso, que no reprocha ni culpa a Birgitte por tener que ejercer él un papel que ha sido asignado históricamente a las mujeres, mientras su esposa es quien se centra y prospera en el ámbito profesional.

Sin embargo, aunque un personaje represente la ruptura de determinados estereotipos, no significa que haya superado todos los mantras del patriarcado. Por ejemplo, al principio de la serie vemos que compara a hombres y mujeres y afirma que «las mujeres no saben ir de farol, ¿por qué te crees que hay tan pocas mujeres que jueguen al póker?», o cuando en el capítulo 5 de la segunda temporada, con respecto a la propuesta de ley sobre cuotas obligatorias del $45 \%$ de mujeres en los consejos de las empresas danesas, comenta: «No me gusta, apesta a intervencionismo» o también que «lo verán como una conspiración femenina».

En este punto es interesante señalar la reflexión de Nuria Varela respecto a la posición de los hombres respecto a la deconstrucción de la masculinidad, para atender a la importancia del personaje de Phillip Christensen: 
La versión dominante de la identidad masculina no constituye una esencia, sino una ideología de poder que tiende a justificar la dominación masculina sobre las mujeres. Además, la identidad masculina se aprende y, por tanto, se puede cambiar. Entonces, si las mujeres llevan décadas comprometidas en deconstruir la feminidad, surgen preguntas inevitables: ¿Por qué tantos varones permanecen en una posición inmovilista? (Varela, 2005: 327).

El principal valor de este personaje es que resulta fundamental para mostrar nuevos ejemplos de organización familiar basados en la igualdad de género. Por eso podemos afirmar que se trata de un personaje masculino que no muestra una posición inmovilista, siguiendo la explicación de Nuria Varela, en cuanto a los roles de género, contribuyendo de esta manera a mostrar en la ficción política modelos de conducta que no reproducen los estereotipos asociados a la masculinidad hegemónica.

\section{Kasper Juul:}

Kasper Juul es la representación del «todo vale» en política. Como jefe de prensa de Birgitte Nyborg no duda en proponer o llevar a cabo prácticas poco éticas, como podemos observar ya desde el principio de la serie cuando roba unas facturas en la casa del asesor del Primer Ministro y luego se las entrega al líder del Partido Laborista.

En cuanto a los atributos mencionados anteriormente sobre rasgos de personalidad estereotípicos, a Kasper Juul se le podría atribuir el talento, la racionalidad, el poder, la agresividad, amante del peligro, duro, individualista, con personalidad fuerte y egoísta.

Podemos ver que Kasper trata a las mujeres de la serie como objetos para mantener relaciones sexuales. En las fiestas o celebraciones políticas le vemos seducir a mujeres jóvenes sin importarle muchas veces siquiera su nombre, muestra de ello es que durante la serie aparecen antiguos ligues que van a saludarle, por ejemplo, y él ni siquiera recuerda quién son. Tampoco le importa mezclar el sexo con el trabajo, pues se acuesta con la secretaria de la Primera Ministra, Sanne (no conocemos el apellido en ningún momento de la serie), o incluso intenta seducir a la ministra de comercio (capítulo 5 de la temporada 1).

Pero cuando las mujeres que aparecen en la trama no son santo de su devoción, rápidamente desaparecen la caballerosidad y la galantería y aparece el odio latente que no tiene ningún reparo en mostrar. Por ejemplo, en el capítulo 5 de la temporada 1, hablando de la ministra de igualdad y asuntos sociales, Pernille Madsen, Kasper le comenta a Birgitte Nyborg que Pernille le recuerda a «una fiesta campera llena de feministas rancias oliendo a monte y apestando a barbacoa. No puedo con ella». Y tras una mirada juiciosa de Birgitte, le replica: «Lo único que digo es que acabará de las primeras en el paredón cuando llegue la revolución», en tono jocoso, bromista.

Con Katrine Fønsmark, con quien mantiene una relación intermitente durante toda la serie, la historia es diferente. Kasper tiene sentimientos reales por Katrine, 
a diferencia de las otras mujeres con las que se acuesta en la serie, y muestra una actitud hacia ella paternalista, posesiva y tóxica.

Podemos ver en algunos puntos de la trama cómo intenta que Katrine Fønsmark haga lo que él piensa que se debe hacer, sin pararse a pensar qué es lo que quiere ella o necesita. Un ejemplo de esto lo vemos en el tercer capítulo de la serie cuando ella descubre que está embarazada y él le insta a abortar, pareciendo que el tema va con él directamente y alegando argumentos como «no puedes dar un vuelco así a tu vida», cuando el bebé ni siquiera es suyo y ella tampoco le ha pedido consejo.

También se inventa un pasado para no revelarle a Katrine qué es lo que le ocurrió de pequeño, de forma que los secretos y las mentiras son una constante en su relación, y Katrine se lo recrimina en repetidas ocasiones. Con respecto a esto, es interesante dejar patente la explicación que hace Nuria Varela en lo referente a la masculinidad y su relación con las conductas de riesgo en el libro Feminismo para principiantes: «En los juegos de niños y en la vida de muchos hombres lo importante es ganar, participar es una vulgaridad. Para ganar hay que aprender a ocultar las propias carencias y evitar la confianza, algo que a los varones se les inculca como peligroso» (Varela, 2005, p.333).

\section{Michael Laugesen:}

Por último vamos a analizar el perfil de Michael Laugesen, que empieza la serie siendo el líder del Partido Laborista y, tras su dimisión, se convierte en redactor jefe del periódico Ekspres. Nos hallamos ante el claro antagonista de esta serie. Tanto en su rol de político como en el de periodista, este personaje está decidido a acabar con Birgitte Nyborg y con su gobierno sea como sea.

Su periódico es la representación del amarillismo en la serie: no tiene problemas en difundir rumores sobre la vida personal de la Primera Ministra y publica en portada que mantiene una relación secreta con su jefe de prensa para desprestigiarla (capítulo 3, primera temporada). Tampoco duda en publicar una fotografía de la ministra de comercio en ropa interior de cuando era más joven y trabajaba de modelo (capítulo 5, temporada 1) cuando está intentando sacar adelante la ley de cuotas del $45 \%$ de mujeres en las empresas de Dinamarca o incluso en obligar a sus subordinadas a escribir un artículo sobre la infidelidad del ministro de exteriores a su mujer con un hombre, treta organizada por él mismo para conseguir presionar al ministro (capítulo 4, temporada 2).

$\mathrm{Su}$ actitud hacia las mujeres denota clara superioridad. En el primer capítulo ya le vemos dirigirse a la líder del Colectivo Solidaridad apelando a su belleza: « ¿Quién dice que no se puede ser de izquierdas e increíblemente hermosa?», o darle una palmada en el culo a la maquilladora del programa en que se retransmite el debate electoral. En el capítulo siguiente le pillan correos electrónicos hablando de una política en los que expresa que «solo verla me pone enfermo, cuando se dirige al Parlamento con su velo de fanática publicitando una religión medieval y antidemocrática».

Respecto a los estereotipos de género relativos a la personalidad de los personajes, Michael Laugessen representaría talento, poder, agresividad, amante del 
peligro, atlético, actúa como líder, duro, individualista, con personalidad fuerte y egoísta. Prácticamente todos.

\section{- Competencia temática}

En cuanto a este ámbito, se suelen reproducir principalmente dos estereotipos: por una parte, se asocia a las mujeres con los llamados temas compasivos (compassion issues) o temas femeninos (feminine issues) que son los temas relacionados con la lucha contra la pobreza, la educación, el arte, el cuidado de la salud; y, por otra parte, se considera que los hombres son más competentes en los llamados temas masculinos (masculine issues) que incluyen cuestiones de economía, empleo, comercio, defensa, asuntos exteriores, control de armas, entre otros. Borgen rompe con ambos.

La ruptura del primer estereotipo va ligada a la trama, pues el hecho de que sea una mujer quien ejerza el rol de Primera Ministra ya lo inhabilita. Pero esto se refuerza siendo habituales situaciones en las que Birgitte Nyborg trata temas que serían considerados como «masculinos». Por ejemplo, en el cuarto capítulo de la primera temporada la vemos inmersa en negociaciones con Estados Unidos para revocar acuerdos llevados a cabo por el anterior gobierno con la administración Bush, negociaciones en las que se tratarán temas como la autonomía de Groenlandia y la seguridad nacional.

También la vemos viajar a Afganistán a visitar a las tropas danesas en el primer capítulo de la segunda temporada o mediar en la guerra entre dos repúblicas africanas ficticias llamadas Kharun del Norte y Kharun del Sur en el capítulo 7 de la segunda temporada.

A pesar de desconocer los nombres de todos los ministros que conforman el gobierno de Birgitte Nyborg, sabemos que la ministra de comercio (cartera considerada masculina) es Henriette Klitgaard, del Partido Moderado, y tras su dimisión, la sustituye Pernille Madsen, quien también forma parte de Comité de Coordinación.

Por lo tanto, podemos afirmar que Borgen también rompe con estos dos estereotipos de género en cuanto a la competencia temática.

\section{- $\quad$ Liderazgo}

Birgitte Nyborg presenta cualidades como líder consideradas estereotípicamente masculinas y también femeninas, y este hecho no hace cuestionar su liderazgo.

Frente a un liderazgo «masculino» caracterizado por la obstinación por el poder, el enfrentamiento o la rivalidad constante, Birgitte Nyborg representa un modo de hacer política basado en la negociación, el dialogo y la transparencia (Padilla Castillo y Sosa Sánchez, 2018), pero también en la asertividad, la racionalidad y la fortaleza, la autonomía personal y la insumisión, rasgos estereotípicamente asociados al género masculino.

Por eso podemos considerar que el liderazgo de Nyborg rompe con un modo «masculino» de hacer política al que estamos habituados a ver especialmente en la 
ficción televisiva, pero tampoco ejerce un estilo de liderazgo «femenino», tomando como base las características arquetípicas ligadas al género femenino en este respecto.

Cuando en el capítulo 5 de la primera temporada Birgitte Nyborg se planta ante Joachim Chrone -uno de los empresarios más importantes de Dinamarca, responsable del 11\% del PIB danés- que amenaza con llevarse sus empresas del país si el gobierno de Nyborg aprueba la ley que obliga a tener un mínimo del $45 \%$ de mujeres en los consejos de las empresas danesas, acierta con que la amenaza de Chrone es un farol, se muestra firme con su decisión y no cede ante el chantaje, como ella misma le expresa a su marido, «acaba ganándole al póker». Aquí podríamos ver esa parte más «masculina» de ejercer el liderazgo.

Sin embargo, también vemos la parte más emocional, la tradicionalmente asociada a las mujeres, especialmente en sus discursos. Cabe destacar el discurso que realiza en el capítulo 10 de la segunda temporada, tras el escándalo mediático al que se ve sometida a causa de tomarse un tiempo de excedencia para cuidar de su hija enferma y la consiguiente polémica que se genera en torno a si las mujeres están capacitadas para gobernar. Con este discurso consigue llegar a la sensibilidad del espectador mostrando su lado más humano, haciendo un repaso por las primeras mujeres políticas que consiguieron un escaño en el Parlamento y acabando con el debate "absurdo», según sus propias palabras, que supone cuestionar en el siglo XXI si las mujeres están preparadas para gobernar un país: «¿Cree realmente algún miembro de este Parlamento que deseaba retirarme y ser ama de casa? Si es así, es que no me conoce en absoluto».

También cabría mencionar el discurso que realiza en el debate electoral televisado (capítulo 1, temporada 1) y que se considera clave de su éxito en las elecciones, en el que habla a los votantes de igual a igual, haciendo alusión a cuestiones relativas a su ámbito privado: «Ahora mi director de campaña está ahí maldiciéndome porque no me he ceñido al discurso. También está enfadado porque no llevo el vestido que él quería, pero por desgracia no me queda bien porque he engordado demasiado».

El liderazgo de Birgitte Nyborg, por lo tanto, presenta un cambio de paradigma, entendiendo que el ejercicio del poder pasa por desplazar los rasgos patriarcales del modo de hacer política.

Sonia Reverter Bañón y María Medina Vicent subrayan, en su análisis «Intersecciones entre liderazgo y feminismo», «la importancia para los feminismos de comprender los posibles procesos de empoderamiento y liderazgo», procesos que nos guíen hacia un «liderazgo más solidario y creativo que sea capaz finalmente de llevarnos a pensar el concepto de política desde otros parámetros nuevos para la convivencia e interrelación social» (Reverter Bañón y Medina Vicent, 2017, p. 9). Esta definición encajaría en el estilo de liderazgo que vemos que Birgitte Nyborg ejerce durante su etapa en el gobierno en Borgen.

Por otra parte, que el personaje de la Primera Ministra presente características o conductas asociadas tradicionalmente al género masculino no se muestra de forma peyorativa en la serie, sino todo lo contrario, por lo que podemos afirmar que la 
perspectiva que se toma no perjudica la imagen de Birgitte Nyborg, ni contribuye a fomentar el llamado doble vínculo que subyace en este estereotipo. «Se la muestra como una política íntegra: da una perfil positivo de una mujer en el poder» (Vélez, 2020, p.90).

Si bien no podemos saber si este efecto también se produce entre el electorado ficticio que juzga el modelo de liderazgo de la Primera Ministra, pues no aparece representado en la serie, sí que podemos ver que algunos personajes critican a Birgitte cuando muestra actitudes consideradas como «masculinas». Un ejemplo se da en el capítulo 5 de la primera temporada, cuando Pernille Madsen (ministra de Igualdad y Asuntos Sociales, forma parte del Partido Laborista) pretende desobedecer las directrices de Birgitte Nyborg y esta se impone: «Soy tu jefa, no vayas, cancélalo», a lo que Pernille responde con tono recriminatorio: "Qué estilo de liderazgo más masculino».

Y respecto al último estereotipo, que hace referencia a que el liderazgo de las mujeres políticas va a menudo parejo al apoyo de un hombre, cabe señalar que si bien es verdad que el personaje de Bent Sejrø, mentor de Birgitte, tiene un peso importante en el desarrollo de la trama y, por supuesto, en la toma de decisiones de la Primera Ministra, también lo es que en ningún momento se define la imagen de Birgitte en función de su relación con Bent.

Birgitte Nyborg escucha y pide consejo a Bent Sejrø en calidad de amigo próximo, con experiencia política y buen criterio, no en calidad de subordinada. De hecho, cuando la Primera Ministra acude a Bent en busca de ayuda, la frase que este más le repite es: «¿Qué opciones tienes?», invitando a la reflexión y no imponiendo su punto de vista. Vemos esto reflejado a lo largo de la serie, y un ejemplo se da en el segundo capítulo de la primera temporada cuando Bent Sejrø le espeta a Birgitte: «¿Qué opinas? Tú eres la jefa». Esto demuestra la esencia de la relación entre ambos. Y que, al final, la decisión última siempre es de Birgitte.

\section{4.- Resultados y discusión}

Tras el estudio realizado podemos concluir que la serie Borgen rompe con los estereotipos de género que son habitualmente asociados a las mujeres políticas en los medios de comunicación, especialmente en las ficciones seriadas, en lo relativo a los cuatro ámbitos analizados: conciliación laboral-familiar, personalidad, competencia temática y liderazgo.

A partir de esta deconstrucción del género en la ficción, podemos desglosar tres valores principales que se extraen de Borgen: el valor educativo y social, el valor comunicativo y el valor político.

En primer lugar, el valor de esta serie a nivel educativo y social radica en que fomenta la igualdad de género y la superación de estereotipos en los ámbitos político, periodístico y familiar. Entendiendo que las ficciones seriadas son productos ampliamente consumidos -más aún con el auge las plataformas de contenidos audiovisuales- y que estas contribuyen en el proceso de creación de identidades individuales y colectivas, el análisis de personajes desde una perspectiva de género es 
también el análisis de modelos de conducta que sirven de base en la construcción identitaria.

La investigación «Gender stereotypes about intellectual ability emerge early and influence children's interests» (Bian, Leslie y Cimpian, 2017), concluyó que las niñas a los seis años ya se sienten menos inteligentes y capaces que los niños de su misma edad. La explicación de que esta creencia sea aprehendida por las niñas de todo el mundo desde tan pronto radica en la asimilación de los estereotipos de género a los que son sometidas desde el momento en el que nacen. Por eso Borgen es tan importante a nivel social, porque propone referentes que necesitamos y de los que carecemos, empezando por su principal protagonista, Birgitte Nyborg, y siguiendo por los demás personajes que la acompañan en la trama y las relaciones que se forjan entre ellos.

Pero la mayor presencia de mujeres en pantalla no es suficiente. El presente estudio también demuestra que esta presencia debe ir acompañada de un enfoque realista, positivo y con perspectiva de género. La investigación llevada a cabo por Tous-Rovirosa y Aran-Ramspott (2017) señaló que:

A pesar de una mayor presencia y protagonismo de los personajes femeninos, aún persiste en las series políticas a un nivel axiológico una construcción de valores que adscribe los personajes femeninos a una esfera más íntima que pública y que presenta variaciones sustanciales en función del sistema político al que se adscribe cada serie: caracterización negativa / ambición como contravalor / belleza física como atributo de poder / soledad presentada como renuncia o abandono de los personajes masculinos, como penalización (Tous-Rovirosa y Aran-Ramspott, 2017: 691).

Sin embargo, como hemos podido apreciar mediante el análisis realizado, esto es algo que no ocurre en Borgen, lo que permite afirmar que la perspectiva que presenta esta serie contribuye a la difusión y normalización de nuevos modelos de conducta basados en la igualdad de género. «La televisión y la cultura audiovisual crea nuestro imaginario, por eso es importante este tipo de personajes que muestran a mujeres fuertes y capaces en roles tradicionalmente no asociados a la mujer» (Vélez, 2020, p.90).

Otro de los valores de esta serie recae en el nivel comunicativo. Como hemos visto, la forma en que los medios representan a las mujeres, y en concreto a las mujeres políticas, acaba repercutiendo también en la construcción de realidades. De hecho, la investigación realizada por Amanda Haraldsson y Lena Wängnerud, «The effect of media sexism on women's political ambition: evidence from a worldwide study», en la que se analizó cómo afecta el sexismo en los medios a la ambición política de las mujeres en 56 países, concluyó que cuanto mayor es el nivel de sexismo en los medios de un país, menor es la proporción de mujeres políticas en dicho país (Haraldsson, A., y Wängnerud, L., 2019). Así pues, teniendo en cuenta la gran repercusión social que puede tener el enfoque que dan los medios de las mujeres políticas, Borgen proporciona una guía a seguir a la hora de realizar productos audiovisuales feministas, tanto en el ámbito informativo como en el del entretenimiento. 
El tercer valor de Borgen estaría asociado al nivel político, pues presenta una hoja de ruta para poder realizar una comunicación política con perspectiva de género. Birgitte Nyborg propone un cambio de paradigma en cuanto al ejercicio del poder, desplazando los rasgos patriarcales del modo de hacer política. El liderazgo que lleva a cabo está basado en la negociación, la transparencia, la asertividad, la racionalidad y la fortaleza, combinando así rasgos asociados, errónea y tradicionalmente, a hombres o mujeres, y difuminando los límites del género en la forma de hacer política.

En definitiva, mediante la realización de este análisis de la serie Borgen hemos podido observar que existe otra forma de representar a las mujeres en las ficciones seriadas y de llevar a cabo una comunicación política que, no solo se aleja, sino que propone una gran disidencia con respecto a los mandatos y expectativas patriarcales. Esto repercute en un producto cultural que presenta una deconstrucción del género en cuanto a la conciliación laboral-familiar, la personalidad, la competencia temática y el liderazgo, planteando así nuevos modelos de conducta, de organización familiar y de concebir la política que abogan por la igualdad de género real.

\section{Referencias bibliográficas}

Belmonte Arocha, Jorge, y Guillamón Carrasco, Silvia (2008). «Co-educar la mirada contra los estereotipos de género en TV». Disponible en http:/ / repositori.uji.es/xmlui/handle/10234/20133 (Fecha de consulta 15/06/2020).

BIAN, Lin, LESLIE, Sarah Jane, y CIMPIAN, Andrei. (2017). «Gender stereotypes about intellectual ability emerge early and influence children's interests». Science, 355(6323), 389-391.

Boukes, Mark, AALbers, Lotte, y ANDERSEN, Kim (2020). «Political fact or political fiction? The agenda-setting impact of the political fiction series Borgen on the public and news media» en Communications, 1.

CARrión DOMínguez, Ángel (2019). «The ones who knock. La inventio narrativa de la quality tv en la edad de oro de las ficciones seriadas. Un estudio tematológico» (Doctoral dissertation, Universitat Autònoma de Barcelona).

CAstells, Manuel (2008). «Comunicación, poder y contrapoder en la sociedad red (I). Los medios y la política» en Telos, 74(1-19).

Fernández García, Nuria (2010). «Framing Hillary Clinton en la Prensa Española: ¿Candidata o mujer?» en Observatorio $\left(\mathrm{OBS}^{*}\right), 4(3)$.

Francisco-Lens, Noelia y Rodríguez-vÁzQuez, Ana Isabel (2020). «La innovación de la Televisión Pública Europea en la oferta audiovisual digital: nuevas plataformas para la Generación Z» en Revista de la Asociación Española de Investigación de la Comunicación, 7(13), 185-212.

GALÁN FAJARDo, Elena (2007) «Construcción de género y ficción televisiva en España» en Comunicar: Revista científica iberoamericana de comunicación y educación, (28), 229-236.

García BeAudoux, Virginia (2014). «Influencia de la televisión en la creación de 
estereotipos de género y en la percepción social del liderazgo femenino. La importancia de la táctica de reencuadre para el cambio social» en Ciencia política, 9(18), 20.

Haraldsson, Amanda y Wängnerud, Lena (2019). «The effect of media sexism on women's political ambition: evidence from a worldwide study» en Feminist Media Studies, 19:4, 525-541, DOI: 10.1080/14680777.2018.1468797.

Hormigos Vaquero, Montserrat y BAllester Buigues, Irene (2020). «La mirada feminista ante el espejo publicitario» en Asparkía. Investigació Feminista. No36, pp. 11-13.

LoIs, Marta y Alonso, Alba (2014). Ciencia politica con perspectiva de género. Madrid: Akal.

MARín RAmos, Esther (2019). Más allá de Bechdel: «The Good Wife, The Good Fight y Orange is the new Black. La imagen de la mujer en las series de televisión feministas» en Universitas Humanística, 87(87).

Padilla Castillo, Graciela y Sosa SÁnchez, Roxana Popelka (2018) «Ruptura de los estereotipos de género en la ficción televisiva sobre el poder político: el caso Borgen» en Revista de Comunicación 'Vivat Academia', (145).

ReVerter-Bañón, Sonia y Medina-Vicent, María (2017). «Intersecciones entre liderazgo y feminismo» en Dossiers feministes, (22), 5-12.

Sierra Caballero, Francisco (2017) «Comunicación y Género. Agendas y cultura de investigación» en Chasqui. Revista Latinoamericana de Comunicación, (135), 09-14.

Tous-Rovirosa, Anna y ARAN-RAMSPOTT, Sue (2017). «Mujeres en las series políticas contemporáneas. ¿Una geografía común de su presencia en la esfera pública?» en El profesional de la información, 26(4), pp. 684-694. https://doi. org/10.3145/epi.2017.jul.12

VArela, Nuria (2005). Feminismo para principiantes, Barcelona: Penguin Random House Grupo Editorial.

VÉlez, Anabel (2020). Mujeres en las series: Fuertes, rebeldes, fantásticas, ganadoras: ellas son la clave. Ma Non Troppo.

Recibido el 22 de junio de 2020

Aceptado el 20 de abril de 2021 BIBLID [1132-8231 (2021): 369-387] 